\title{
Prospective Spatio-Temporal and Temporal Cluster Detection by Salmonella Serotype
}

\author{
Eric R. Peterson*, Vasudha Reddy, HaeNa Waechter, Lan Li, Kristen Forney and \\ Sharon K. Greene
}

New York City Department of Health and Mental Hygiene, Queens, NY, USA

\section{Objective}

To prospectively identify serotype-specific clusters of salmonellosis in New York City (NYC).

\section{Introduction}

Nontyphoidal Salmonella, consisting of $>2,500$ distinct serotypes, is the leading bacterial agent of foodborne illness in the U.S., causing an estimated 1 million infections per year. ${ }^{1}$ In NYC, interviews of all case-patients $(\mathrm{N} \approx 1,100$ annually) are attempted to support outbreak investigation and control. Salmonella clusters in NYC are typically identified either by notification from PulseNet, CDC, or other health departments or by a weekly analysis using the historical limits method. More systematic and timely cluster detection could inform resource prioritization and improve the effectiveness of public health interventions. We initiated daily analyses in May 2015 to detect spatio-temporal clusters by serotype among cases since February 23. In July 2015, an analysis was added to detect purely temporal clusters among cases since May 1.

\section{Methods}

Salmonella isolates for NYC residents are serotyped by the city and state public health laboratories and reported to the Bureau of Communicable Disease of the NYC Department of Health and Mental Hygiene. An automated process assigned a standardized serotype to each salmonellosis case. Each case's residential address at time of report was geocoded in near real-time and assigned a census tract. Each case was also assigned an "event date," representing the illness onset date.

We used the prospective space-time permutation scan statistic to detect and evaluate clusters. ${ }^{2}$ A spatio-temporal analysis, with a maximum spatial size of $50 \%$ of observed episodes, was run for each serotype with a case reported in the past 60 days. A temporal analysis was run for all serotypes, replacing "space" in the space-time permutation scan statistic with "serotype" and setting the maximum spatial size to $0 .{ }^{3}$ For all analyses, the temporal cluster size range was 2 to 60 days. A 14-day lag was implemented to allow for data accrual. The baseline period was 1.5 years. Monte Carlo simulations $(\mathrm{N}=999)$ were used to determine statistical significance. Automated analyses were run each morning using Microsoft Task Scheduler, SAS 9.2, and SaTScan 9.4.1.

Any cluster with a recurrence interval (RI) $\geq 45$ days was summarized in a map and linelist. Foodborne disease epidemiologists assessed clusters to determine if cases were linked by a common exposure and/or by pulsed-field gel electrophoresis (PFGE) of isolates.

\section{Results}

With event dates February 23-July 29, 2015, 391 Salmonella cases of 48 serotypes were reported, and 7 spatio-temporal clusters were identified for 5 serotypes. With event dates May 1-July 29, 254 cases of 42 serotypes were reported, and 5 temporal clusters were identified for 5 serotypes. The most unusual clusters were citywide temporal clusters of $S$. Poona ( $\mathrm{N}=9, \mathrm{RI}=14.5$ years), $S$. Oranienburg $(\mathrm{N}=4, \mathrm{RI}=4.3$ years), and $S$. Berta $(\mathrm{N}=13, \mathrm{RI}=3.0$ years $)$, and a spatio-temporal $S$. Heidelberg cluster ( $\mathrm{N}=5, \mathrm{RI}=456$ days), which at the time of signaling corresponded to clusters already identified and under investigation. A cluster of $S$. Muenchen, first identified by our temporal ( $\mathrm{RI}=67$ days), then by our spatio-temporal analyses $(\mathrm{RI}=59$ days) 8 days later, ultimately included 6 cases whose isolates were indistinguishable by PFGE. The investigation is ongoing.

\section{Conclusions}

In the first few months of prospective, automated cluster detection analyses by serotype, a manageable number of clusters were detected. The purely temporal analysis was sensitive to rare serotypes. All clusters either prompted new investigations or corresponded to ongoing investigations, complementing NYC's existing enhanced Salmonella surveillance system. These methods could be useful for other health departments to adopt for primary or confirmatory cluster detection.

\section{Keywords}

Salmonella; foodborne illness; outbreak detection

\section{Acknowledgments}

Deborah Kapell and Alison Levin-Rector contributed to the SAS code; Ana Maria Fireteanu contributed to standardizing serotyping data; Public Health Laboratory colleagues performed serotyping and PFGE; and Martin Kulldorff provided guidance.

\section{References}

1. Scallan E, et al. Foodborne illness acquired in the United States-major pathogens. Emerg Infect Dis. 2011;17:7-15.

2. Kulldorff M, et al. A space-time permutation scan statistic for disease outbreak detection. PLoS Medicine. 2005;2:e59.

3. Huang SS, et al. Automated detection of infectious disease outbreaks in hospitals: a retrospective cohort study. PLoS Medicine. 2010;7:e1000238.

*Eric R. Peterson

E-mail: epeterson@health.nyc.gov 